\title{
The virial relation for compact Q-balls in the complex signum-Gordon model
}

\author{
Huawen Wang Hongbo Cheng* \\ ${ }^{\dagger}$ Department of Physics, East China University of Science and Technology, \\ Shanghai 200237, China \\ The Shanghai Key Laboratory of Astrophysics, \\ Shanghai 200234, China
}

\begin{abstract}
In this work the properties of Q-balls in the complex signum-Gordon model in $d$ spatial dimensions is studied. We obtain a general virial relation for this kind of Qball in the higher-dimensional spacetime. We compute the energy and radii of Q-ball with V-shaped field potential as a function of spatial dimensionality and a parameter defining the model potential energy density to show that this kind of Q-balls can also be survive stably in the high-dimensional spacetime.
\end{abstract}

PACS number(s): 11.27.+d, 11.10.Lm, 98.80.Cq

*E-mail address: hbcheng@sh163.net

${ }^{\dagger} 021-64703389(\mathrm{H}) ; 13661864435(\mathrm{M})$ 
The nontopological solitons possess a conserved Noether charge because of a symmetry of the Lagrangian of system in contrast to the topological charge resulting from the spontaneous symmetry breaking in the case of topological defects $[1,2]$. As nontopological solitons Q-balls appear in extended localized solutions of models with certain self-interacting complex scalar field [3]. Their stability is associated with their charge $Q$ and that their mass is smaller than the mass of a collection of scalar fields. The unbroken continuous globally symmetry like $U(1)$ is also possessed. The standard models have the smooth potentials near their absolute minima, so they hold exponential tails and interact with each other. Such solitons are physically universal and have been studied in several subjects like dark matter [4-6], condensed matter physics [7] and so on. The Q-balls have also attracted much attentions in cosmology [8-11]. The Boson stars can be considered as solutions of complex scalar field models couple to gravity [12-19]. The general nontopological solitons have also been explored in de Sitter and anti de Sitter spacetimes respectively [20, 21].

There is plenty of motivation to research on the nontopological soliton models in the higherdimensional spacetimes. The issue of higher-dimensional spacetime can help us to unify the interactions in nature with extra compactified dimensions and to solve the hierarchy problem with an additional warped dimension [22-25]. The signatures of these extra dimensions may be explored in various directions. The properties of high-dimensional world also dominate the structure and the stability of various kinds of nontopological soliton models including Q-balls. We can search for the dependence of Q-balls configuration and energy subject to a finite charge on the spatial dimensions. Q-balls may survive well in a precompactification world while modify the structure of the vacuum. Studying the Q-balls in the Universe with more than four dimensions certainly help us to probe the background with additional spatial dimensions.

A new kind of interesting nontopological solitons was put forward [26] and some important efforts have been contributed [27]. The so-called complex signum-Gordon model has the field potential with sharp bottom rather than wholely smooth one. Further the V-sharped field potential can be denoted as $U(\phi)=\lambda|\phi|$, where $\lambda$ is positive constant and $\phi$ is a complex scalar field. The inverted-conical-shaped potentials are certainly different from the case of other nontopological solitons with potential involving higher order of fields. There exist the Q-balls in the complex signum-Gordon model, which are stationary and whose energy is finite while their scalar field, energy density and charge density approach to the zero outside the ball region. It should be pointed out that these Q-ball solutions are simpler than the others. The boson stars and black holes in scalar electrodynamics with a $\mathrm{V}$-shaped scalar potential are considered [28]. The spacetime around this kind of boson stars consists of a Schwarzschild-type black hole in the interior and a Reissner-Nordstrom-type spacetime in the exterior appears.

It is significant to study the compact Q-balls in the complex signum-Gordon model in higherdimensional spacetimes by means of virial theorem. The task for exploring the Q-balls with Vshaped field potential in the four-dimensional background [26-28], but the properties of this kind of Q-balls in the higher-dimensional spacetimes have not studied in detail. A generalized virial relation 
for Q-balls involving general smooth potentials like $V\left(\phi \phi^{+}\right)=\sum_{n=1}^{\infty} a_{n}\left(\phi \phi^{+}\right)^{n}$ with constants $a_{n}$ and integer $n$ in $d$ spatial dimensions was obtained and how spatial dimensionality affects some of the key properties of Q-balls such as their energy, minimal charge and size was also declared [29]. We will study the same kind of Q-balls in the high-dimensional spaacetime by means of the method from Ref. [29]. We should utilize the virial theorem to bring about the necessary conditions on the complex signum-Gordon model while find the model's conserved charge $Q$ and energy associated with the dimensionality and the parameters in the potential.

In this paper we discuss the Q-balls in the complex signum-Gordon model in the world with arbitrary dimensions carefully. We present a d-dimensional virial relation for this kind of Q-balls and the relation will recover to be the Derrick's theorem when $Q=0$. We study these Q-balls to estimate their properties in the case of large charge and radius and small ones respectively. Our results are listed in the end.

Here we consider the complex signum-Gordon model with Lagrangian density as follow,

$$
\mathcal{L}=\partial_{\mu} \phi^{+} \partial^{\mu} \phi-V\left(\phi \phi^{+}\right)
$$

where $\phi$ is a complex scalar field in $(d+1)$-dimensional Minkowski spacetime. The index $\mu=$ $0,1,2, \cdots, d$ and the signature is $(+,-,-, \cdots)$. The potential is assumed to be $V\left(\phi \phi^{+}\right)=\lambda|\phi|$ with a global minimum at $\phi=0$ and the coupling constant $\lambda>0$. As Q-balls this model is nonperturbative excitation about this global vacuum state carring a net particle number named charge $Q$ which is conserved. The condition that the energy of the Q-ball $E_{Q}$ is smaller than $Q m_{\phi}$ which is energetically preferred to keep its own stability. The Lagrangian of the complex signumGordon model has a conserved $U(1)$ symmetry under the global transformation $\phi(x) \longrightarrow e^{i \alpha} \phi(x)$. The associated conserved current should be $j^{\mu}=-i\left(\phi^{+} \partial^{\mu} \phi-\phi \partial^{\mu} \phi^{+}\right)$and the corresponding conserved charge is given by $Q=\int d^{d} x j^{0}$. We introduce the ansatz for configurations with lowest energy,

$$
\phi(x)=\frac{1}{\sqrt{2}} \Phi(\mathbf{x}) e^{i \omega t}
$$

Here the field $\Phi(\mathbf{x})$ can be taken to be spherically symmetry and $\{\mathbf{x}\}$ represent the spatial components of coordinates. The field equation can be obtained,

$$
\left(\nabla_{d}^{2}+\omega^{2}\right) \Phi-\frac{\lambda}{2} \frac{\Phi}{|\Phi|}=0
$$

According to Ref. [29] and from Lagrangian density (1) and ansatz (2), the energy for a field configure in a $(d+1)$-dimensional spacetime can be obtained,

$$
E[\Phi]=\int d^{d} x\left[\frac{1}{2}\left(\nabla_{d} \Phi\right)^{2}+\frac{1}{2} \omega^{2} \Phi^{2}+V\left(\Phi^{2}\right)\right]
$$

We can also denote the energy in terms of the conserved charge $Q$ as follow, 


$$
E_{Q}[\Phi]=\frac{1}{2} \frac{Q^{2}}{\left\langle\Phi^{2}\right\rangle}+\left\langle\frac{1}{2}\left(\nabla_{d} \Phi\right)^{2}\right\rangle+\langle V\rangle
$$

where $\langle\cdots\rangle=\int \cdots d^{d} x$. In order to obtain the virial relation we scale the spatial variable $x \longrightarrow \alpha x$ and impose the invariance of energy under scale transformation $\left.\frac{\partial E}{\partial \alpha}\right|_{\alpha=1}=0$. As a generalization of Derrick's theorem the virial relation for Q-balls in a spacetime with arbitrary dimensionality and written as,

$$
d\langle V\rangle=(2-d)\left\langle\frac{1}{2}\left(\nabla_{d} \Phi\right)^{2}\right\rangle+\frac{d}{2} \frac{Q^{2}}{\left\langle\Phi^{2}\right\rangle}
$$

It should be pointed that the Derrick's theorem can be recovered for $Q=0$. Further the absolute lower bound for Q-balls to be a preferred energy state is shown as $Q^{2} \geq \frac{2(d-2)}{d}\left\langle\Phi^{2}\right\rangle\left\langle\frac{1}{2}\left(\nabla_{d} \Phi\right)^{2}\right\rangle$ because of $\langle V\rangle \geq 0$. Combining Eq. (4)-(6), we express the energy density as,

$$
\frac{E}{Q}=\omega\left(1+\frac{1}{d-2+d \frac{\langle V\rangle}{\left\langle\frac{1}{2}\left(\nabla_{d} \Phi\right)^{2}\right\rangle}}\right) \leq m_{\phi}
$$

Only the Q-balls satisfying the condition (7) are stable instead of dispersing. Here we choose,

$$
\langle V\rangle=\frac{\lambda}{\sqrt{2}} \int d^{d} x|\Phi|
$$

then the formulae mentioned above can be utilized to explore the compact Q-balls of complex signum-Gordon model in higher-dimensional world analytically and the nonlinear field equation needs not be solved.

Now in $(d+1)$-dimensional spacetimes we work on the complex-signum-Gordon-type Q-balls which are characterized by large charge and radius. At first we utilize the Coleman issue [3] to explore the large Q-balls. We choose the field to be a step function which is equal to be a constant denoted as $\Phi_{c}$ within the ball and vanishes out side the ball's volume $v$. The step-function type field leads $\left\langle\Phi^{2}\right\rangle=\Phi_{c}^{2} v, \nabla_{d} \Phi=0$ and $\langle V\rangle=\frac{\lambda}{2}\left|\Phi_{c}\right| v$. From Eq.(5) the model energy is

$$
E=\frac{1}{2} \frac{Q^{2}}{\Phi_{c}^{2} v}+\frac{\lambda}{\sqrt{2}}\left|\Phi_{c}\right| v
$$

Having extremized the expression (9) with respect to the volume $v$, we obtain the minimum energy and the condition for Q-ball's stability as follow,

$$
\frac{E_{\min }}{Q}=2^{\frac{1}{4}}\left(\frac{\lambda}{\Phi_{c}}\right)^{\frac{1}{2}}<m_{\phi}
$$

The complex signum-Gordon Q-ball can exist if the model parameters are adjusted reasonably.

In order to describe the large Q-balls in the complex signum-Gordon model, we introduce the field profile,

$$
\Phi(r)=\left\{\begin{array}{cc}
\Phi_{c} & r<R \\
\Phi_{c} e^{-\alpha(r-R)} & r \geq R
\end{array}\right.
$$


where $\alpha$ is a variational parameter. This kind of function keeps the scalar field to be a constant like $\Phi_{c}$ within an area and not to drop to the zero in the larger region whose size is larger than $R$. According to large-Q-ball ansatz (11), the energy of model reads,

$$
\begin{aligned}
E= & \frac{1}{2} \frac{Q^{2}}{\left\langle\Phi^{2}\right\rangle}+\frac{1}{2}\left\langle\left(\nabla_{d} \Phi\right)^{2}\right\rangle+\frac{\lambda}{\sqrt{2}}\langle|\Phi|\rangle \\
= & \frac{1}{2} \omega^{2} \frac{c_{d}}{d} \Phi_{c}^{2} R^{d}+\frac{1}{2} \omega^{2} c_{d} \Phi_{c}^{2}(d-1) ! \sum_{k=0}^{d-1} \frac{1}{k !} \frac{1}{(2 \alpha)^{d-k}} R^{k} \\
& +\frac{1}{2} c_{d} \Phi_{c}^{2} \alpha^{2}(d-1) ! \sum_{k=0}^{d-1} \frac{1}{k !} \frac{1}{(2 \alpha)^{d-k}} R^{k} \\
& +\frac{\lambda}{\sqrt{2}} \frac{c_{d}}{d} \Phi_{c} R^{d}+\frac{\lambda}{\sqrt{2}} c_{d} \Phi_{c}(d-1) ! \sum_{k=0}^{d-1} \frac{1}{k !} \frac{1}{\alpha^{d-k}} R^{k}
\end{aligned}
$$

while the conserved charge is obtained,

$$
\begin{aligned}
& Q=\omega \int d^{d} x \Phi^{2} \\
& =\omega \frac{c_{d}}{d} \Phi_{c}^{2} R^{d}+\omega c_{d} \Phi_{c}^{2}(d-1) ! \sum_{k=0}^{d-1} \frac{1}{k !} \frac{1}{(2 \alpha)^{d-k}} R^{k}
\end{aligned}
$$

which is the same as that of Ref. [29], here

$$
c_{d}=\frac{2 \pi^{\frac{d}{2}}}{\Gamma\left(\frac{d}{2}\right)}
$$

The conserved charge is able to replace the frequency $\omega$. We just keep the dominant terms in the expressions for the energy and this approximation is accurate enough for large Q-balls. Combining the expression for $Q$ in Eq. (13), we find

$$
E_{Q} \leq \frac{Q^{2} d}{2 c_{d} \Phi_{c}^{2}} R^{-d}+\frac{c_{d} b}{\sqrt{2} d} R^{d}+\left(\frac{c_{d} \alpha \Phi_{c}^{2}}{4}+\frac{c_{d} b}{\sqrt{2} \alpha}\right) R^{d-1}
$$

where

$$
b=\lambda \Phi_{c}
$$

It is clear that the model energy depends on the variables $R$ and $\alpha$ not belonging to the model parameters. We extremise the energy expression (15) with respect to $R$ and $\alpha$ respectively. By means of performance $\left.\frac{\partial E}{\partial R}\right|_{R=R_{c}}=0$ we find the equation that the critical radius $R_{c}$ satisfies,

$$
-\frac{Q^{2} d^{2}}{2 c_{d} \Phi_{c}^{2}}+\frac{c_{d} b}{\sqrt{2}} R^{2 d}+(d-1)\left(\frac{c_{d} \Phi_{c}^{2} \alpha}{4}+\frac{c_{d} b}{\sqrt{2} \alpha}\right) R^{2 d-1}=0
$$

The approximate solution to Eq. (17) is, 


$$
R_{c} \approx 2^{-\frac{1}{4 d}}\left(\lambda \Phi_{c}\right)^{-\frac{1}{2 d}}\left(\frac{Q d}{c_{d} \Phi_{c}}\right)^{\frac{1}{d}}-\frac{d-1}{\sqrt{2} d}\left(\frac{\alpha \Phi_{c}}{4 \lambda}+\frac{1}{\sqrt{2} \alpha}\right)
$$

and the solution is valid for large Q-balls. We can also impose the condition $\left.\frac{\partial E_{Q}}{\partial \alpha}\right|_{\alpha=\alpha_{c}}=0$ into Eq. (15) to find,

$$
\alpha_{c}=2^{\frac{3}{4}}\left(\frac{\lambda}{\Phi_{c}}\right)^{\frac{1}{2}}
$$

Substituting the results (18) and (19) into Eq. (15), we obtain the extremised expression for the energy of large Q-ball,

$$
\frac{\left.E_{Q}\left[\Phi_{c}\right]\right|_{R_{c}, \alpha_{c}}}{Q} \approx 2^{\frac{1}{4}}\left(\frac{\lambda}{\Phi_{c}}\right)^{\frac{1}{2}}\left(1+\xi_{c} Q^{-\frac{1}{d}}\right)
$$

where

$$
\xi_{c}=2^{\frac{1}{4}\left(\frac{1}{d}-3\right)} c_{d}^{\frac{1}{d}}\left(\frac{d}{\sqrt{\lambda}}\right)^{1-\frac{1}{d}} \Phi_{c}^{\frac{1}{2}\left(1+\frac{3}{d}\right)}
$$

Having compared the results in Eq. (20) with those in Eq. (10), we also declare that the lower bound on the energy per one particle in large Q-balls is larger than that of Coleman approach. It should be pointed out that $\frac{\left.E_{Q}\left[\Phi_{c}\right]\right|_{R_{c}, \alpha_{c}}}{Q}$ is finite within the context of large radius and conserved charge. We plot the dependence of the minimum energy per unit charge of complex-signum-Gordontype Q-ball on the charge with $\lambda=1$ and $\Phi_{c}=1$ for different spatial dimensions in Fig. 1 and show that the minimum energy per unit charge is a decreasing function of the charge. As the charge $Q$ becomes extremely large, $\frac{\left.E_{Q}\left[\Phi_{c}\right]\right|_{R_{c}, \alpha_{c}}}{Q}$ will approach to the value which is just associated with the model parameters no matter how high the dimensionality is. The Q-ball will possess the larger $\frac{\left.E_{Q}\left[\Phi_{c}\right]\right|_{R_{c}, \alpha_{c}}}{Q}$ in the higher-dimensional world.

We start to discuss the small Q-balls in the signum-Gordon model in the $(d+1)$-dimensional spacetimes. The small Q-balls with radii $R \geq m_{\phi}^{-1}$ can not be described well by means of thinwall approximation. We introduce a Gaussian ansatz in order to consider the small Q-balls in the complex signum-Gordon model,

$$
\Phi(\mathbf{x})=\Phi_{c} e^{-\frac{r^{2}}{R^{2}}}
$$

Substituting the ansatz (22) into the expression (5), we obtain the small Q-ball energy as follow,

$$
\begin{aligned}
& E=\int d^{d} x\left[\frac{1}{2} \omega^{2} \Phi^{2}+\frac{1}{2}\left(\nabla_{d} \Phi\right)^{2}+V\left(\Phi^{2}\right)\right] \\
& =\left(\frac{\pi}{2}\right)^{\frac{d}{2}}\left[\frac{1}{2}\left(\frac{2}{\pi}\right)^{d} \frac{Q^{2}}{\Phi_{c}^{2}} R^{-d}+\frac{d}{2} \Phi_{c}^{2} R^{d-2}+2^{\frac{d-2}{2}} \lambda\left|\Phi_{c}\right| R^{d}\right]
\end{aligned}
$$

The energy for small Q-ball in the signum-Gordon model just contains the dominant terms. By extremizing the expression for the energy with respect to $R$ like $\left.\frac{\partial E_{Q}}{\partial R}\right|_{R=R_{c}}=0$ we obtain the equation for the critical radius $R_{c}$ as, 


$$
R_{c}^{2 d}+\frac{d-2}{d} \frac{d}{2^{\frac{d+1}{2}}} \frac{\Phi_{c}}{\lambda} R_{c}^{2 d-2}-\frac{2^{\frac{d-1}{2}}}{\pi^{d}} \frac{Q^{2}}{\lambda} \frac{1}{\left|\Phi_{c}\right|^{3}}=0
$$

Similarly the approximate solution reads,

$$
R_{c} \approx R_{0}\left(1-\frac{d-2}{d} \frac{1}{2^{\frac{d+3}{2}}} \frac{\Phi_{c}}{\lambda} \frac{1}{R_{0}^{2}}\right)
$$

where

$$
R_{0}=\left(\frac{2^{\frac{d-1}{2}}}{\pi^{d}} \frac{Q^{2}}{\lambda\left|\Phi_{c}\right|^{3}}\right)^{\frac{1}{2 d}}
$$

According to the critical radius the energy can be expressed as

$$
\frac{\left.E\left[\Phi_{c}\right]\right|_{R=R_{c}}}{Q}=2^{\frac{d+1}{4}}\left(\frac{\lambda}{\Phi_{c}}\right)^{\frac{1}{2}}\left[1+\frac{\eta_{c} Q^{-\frac{2}{d}}}{2}-\frac{(d-2)^{2}}{4 d^{2}} \eta_{c}^{2} Q^{-\frac{4}{d}}\right]
$$

where

$$
\eta_{c}=\frac{d}{2^{\frac{d}{2}-\frac{1}{2 d}+1}} \pi \lambda^{\frac{1-d}{d}} \Phi_{c}^{\frac{3}{d}+1}
$$

The stability of small Q-balls can be held according to Eq. (27). The energy over charge is finite while the model parameters can be adjusted. The powers of charge $Q$ in the terms are negative, so the model energy per unit charge will not be divergent as the charge becomes larger. In Fig. 2 , for simplicity we also choose $\lambda=1$ and $\Phi_{c}=1$ without losing generality and we show that the minimum energy per unit charge $\frac{\left.E\left[\Phi_{c}\right]\right|_{R=R_{c}}}{Q}$ also decreases as the charge increases in the case of small charge and radius in the background with different spatial dimensions. The higher dimensionality will lead the larger $\frac{\left.E\left[\Phi_{c}\right]\right|_{R=R_{c}}}{Q}$. Certainly here the energy over charge is smaller than that in the case of large ones.

In this work we investigate the compact Q-balls in the complex signum-Gordon model in the spacetime with arbitrary dimensionality. The Q-ball properties associated with the background structure may help us to further investigate our world. This kind of nontopological model is simple but is enough to contain the properties of Q-balls. We search for the approximate and acceptable analytical description for characteristics of the compact Q-balls in the complex signum-Gordon model instead of solving the nonlinear field equations numerically. We find the virial relation for these kinds of Q-balls. We study the model with V-shaped field potential in the case of large Q-balls and small ones. We obtain the approximate analytical expressions to show the Q-balls' energy and radii depending on the spatial dimensionality and the parameters belonging to the complex signum-Gordon model. The minimum energy per unit charge of the system decreases to a quantity depending on the system parameters and dimensionality. It should be pointed out that the accuracy of the approximate analytical expressions can be acceptable. 


\section{Acknowledgement}

This work is supported by NSFC No. 10875043 and is partly supported by the Shanghai Research Foundation No. 07dz22020. 


\section{References}

[1] Lee T D and Pang Y 1992 Phys. Rep. 221251

[2] Friedberg R, Lee T D and Pang Y 1987 Phys. Rev. D 353658

[3] Coleman S 1985 Nucl. Phys. B 262263

[4] Kusenko A and Shaposhnikov M E 1998 Phys. Lett. B 41846

[5] Kusenko A and Steinhardt P J 2001 Phys. Rev. Lett. 87141301

[6] Mielke E and Schunck F 2002 Phys. Rev. D 66023503

[7] Bunkov Y M and Volovik G E 2007 Phys. Rev. Lett. 98265302

[8] Dvali G R, Kusenko A and Shaposhnikov M E 1998 Phys. Lett. B 41799

[9] Kusenko A, Kuzmin V, Shaposhnikov M E and Tinyakov P G 1998 Phys. Rev. Lett. 803185

[10] Cecchini S et. al. 2008 Eur. Phys. J. C 57525

[11] Takenaga Y et. al. 2007 Phys. Lett. B 64718

[12] Kaup D J 1968 Phys. Rev. 1721331

[13] Jetzer P 1992 Phys. Rep. 220163

[14] Schunck F E and Mielke E 2003 Class. Quantum Grav. 20 R301

[15] Schunck F E and Mielke E 1998 Phys. Lett. A 249389

[16] Colpi M, Shapiro S L and Wasserman I 1986 Phys. Rev. Lett. 572485

[17] Kleihaus B, Kunz J and List M 2005 Phys. Rev. D 72064002

[18] Kleihaus B, Kunz J, List M and Schaffer I 2008 Phys. Rev. D 77064025

[19] Brihaye Y and Hartmann B 2009 Phys. Rev. D 79064013

[20] Cheng H and Gu Z 2005 J. East China Univ. Sci. Technol. 31509

[21] Cheng H and Gu Z 2007 J. East China Univ. Sci. Technol. 33294

[22] Kaluza T 1921 Sitz. Preuss. Akad. Wiss. Phys. Math. K 1966

[23] Klein O 1926 Z. Phys. 37895

[24] Randall L and Sundrum R 1999 Phys. Rev. Lett. 833370

[25] Randall L and Sundrum R 1999 Phys. Rev. Lett. 834690 
[26] Arodz H, Klimas P and Tyranowski T 2005 Acta Phys. Pol. B 363861

[27] Arodz H and Lis J 2008 Phys. Rev. D 77107702

[28] Arodz H and Lis J 2009 Phys. Rev. D 79045002

[29] Gleiser M and Thorarinson J 2006 Phys. Rev. D 73065008 


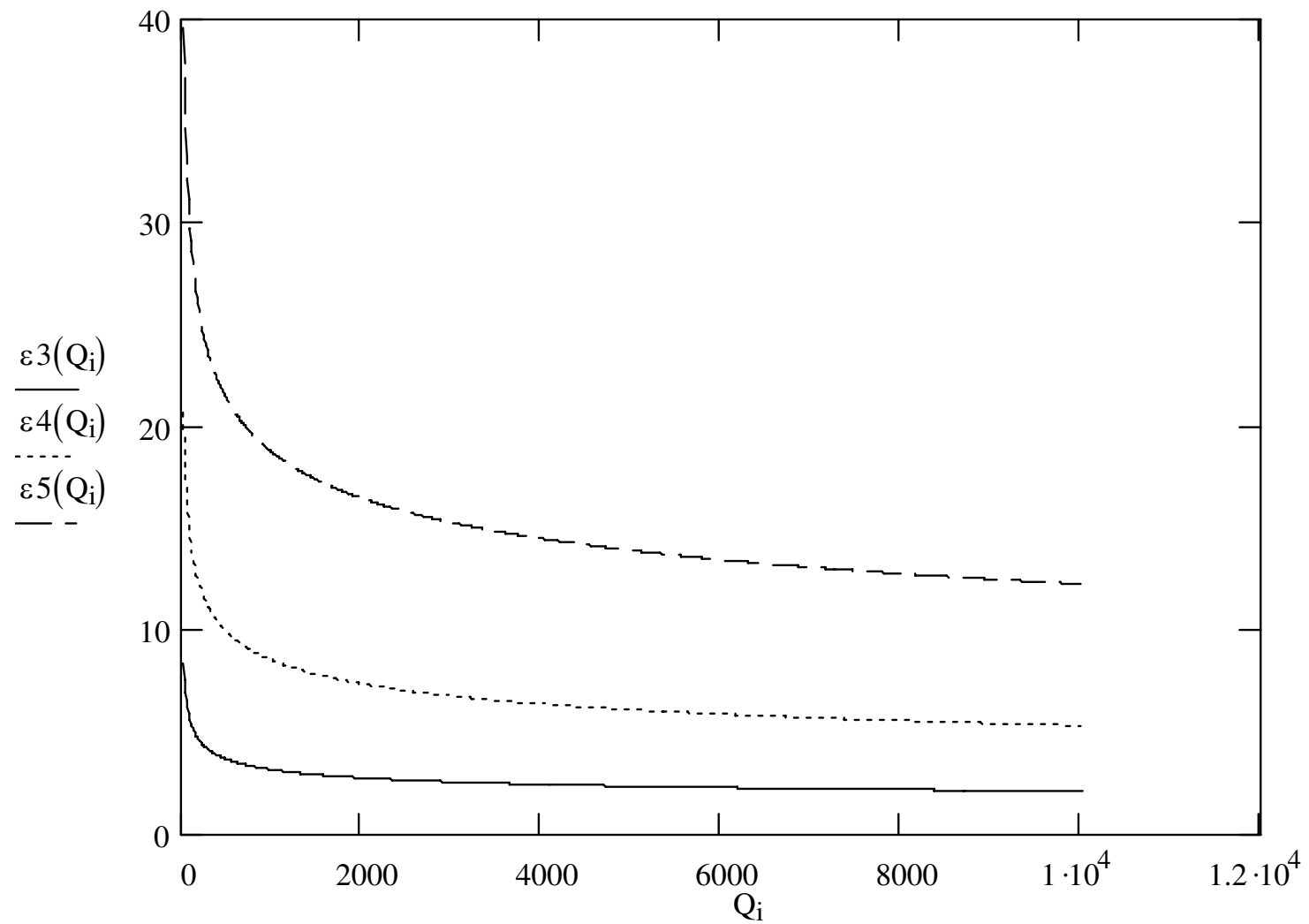

Figure 1: The solid, dot and dashed curves of the minimum energy per unit charge of large Q-balls in the complex signum-Gordon model as functions of charge $Q$ for spatial dimensions $d=3,4,5$ respectively with $\lambda=1$ and $\Phi_{c}=1$. 


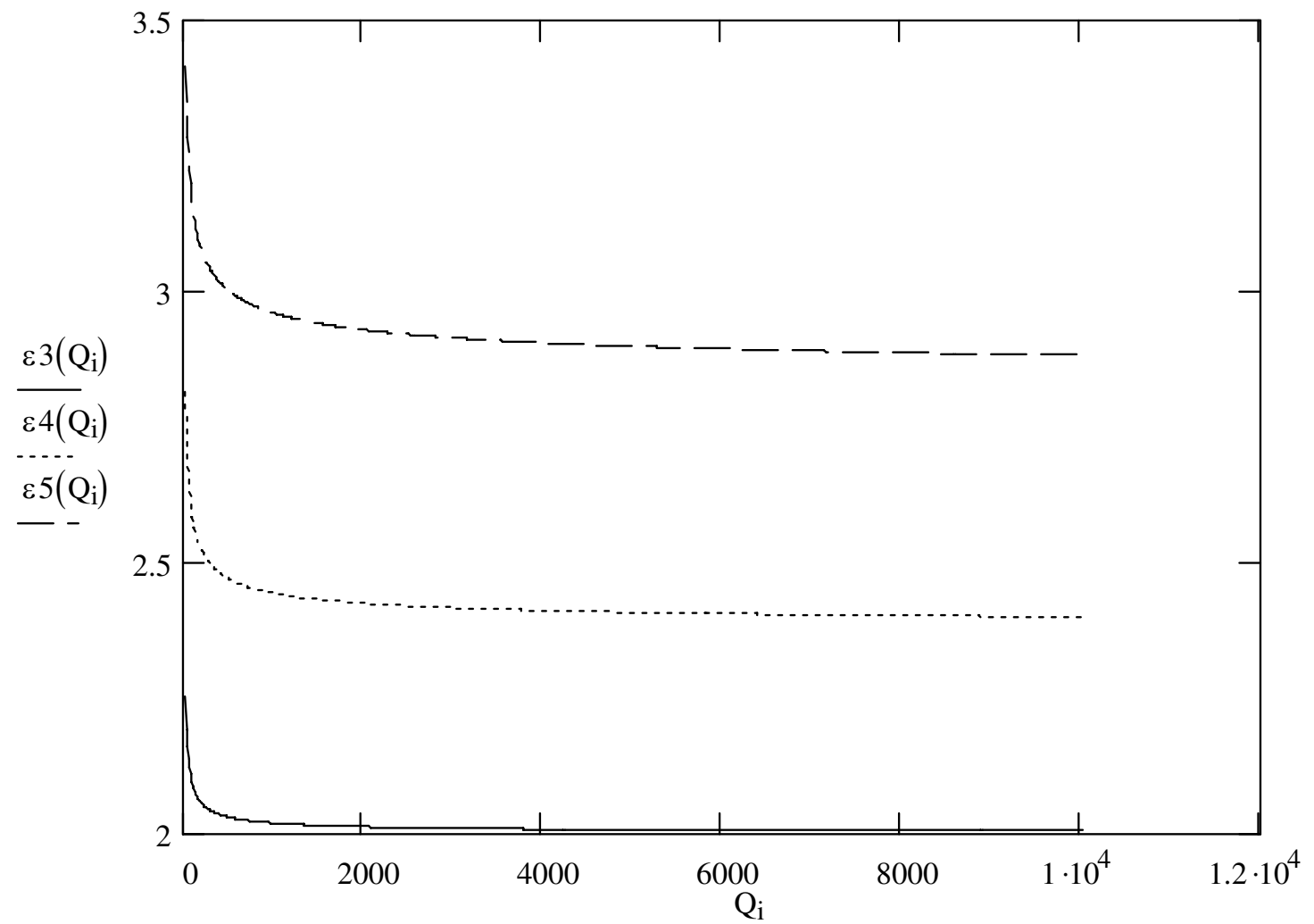

Figure 2: The solid, dot and dashed curves of the minimum energy per unit charge of small Q-balls in the complex signum-Gordon model as functions of charge $Q$ for spatial dimensions $d=3,4,5$ respectively with $\lambda=1$ and $\Phi_{c}=1$. 